\title{
Sheaf fuzzy problems for functional differential equations
}

\author{
Phan Van Tri ${ }^{1}$, Ngo Van $\mathrm{Hoa}^{2 *}$ and Nguyen Dinh $\mathrm{Phu}^{3}$
}

${ }^{\text {*Correspondence: }}$

ngovanhoa_clt@yahoo.com

${ }^{2}$ Division of Computational

Mathematics and Engineering,

Institute for Computational Science,

Ton Duc Thang University, Ho Chi

Minh, Vietnam

Full list of author information is

available at the end of the article

\begin{abstract}
In this paper, we present the studies on two kinds of solutions to fuzzy functional differential equations (FFDEs) and sheaf fuzzy functional differential equations (SFFDEs). The different types of solutions to FFDEs and SFFDEs are generated by the usage of generalized Hukuhara derivative concepts of fuzzy derivative in the formulation of a differential problem. Some examples are given to illustrate these results.
\end{abstract}

Keywords: fuzzy sets; fuzzy functional differential equations; generalized Hukuhara derivative; sheaf fuzzy problems

\section{Introduction}

The study of fuzzy differential equations (FDEs) forms a suitable setting for mathematical modeling of real-world problems in which uncertainties or vagueness pervade. Most practical problems can be modeled as FDEs $[1,2]$. There are several approaches to the study of FDEs. One popular approach is based on H-differentiability for fuzzy number value functions. Under this setting, mainly the existence and uniqueness of the solution of a FFDE are studied (see, e.g., [3-6]). However, this approach has the disadvantage that it leads to solutions which have an increasing length of their support. Recently, Bede and Gal [7] solved the above mentioned approach under strongly generalized differentiability of fuzzy-number-valued functions. In this case the derivative exists and the solution of a fuzzy differential equation may have decreasing length of the support, but the uniqueness is lost. Other researchers have proposed several approaches to the solutions of FDEs (e.g., $[3,8-15])$. Therefore, our point is that the generalization of the concept of $\mathrm{H}$-differentiability can be of great help in the dynamic study of fuzzy functional differential equations and sheaf fuzzy problems for fuzzy functional differential equations. Recently, several works, e.g., [4, 9, 16-20], studied fuzzy differential equations and fuzzy integro-differential equations, fractional fuzzy differential equations, and some methods for solving fuzzy differential equations [21,22] were proposed.

In [10], author considered the fuzzy functional differential equation

$$
\begin{cases}x^{\prime}(t)=f\left(t, x_{t}\right), & t \geq t_{0}, \\ x(t)=\varphi\left(t-t_{0}\right) \in E^{d}, & t_{0} \geq t \geq t_{0}-\sigma,\end{cases}
$$

where $f:[0, \infty) \times C_{\sigma} \rightarrow E^{d}$ and the symbol ' denotes the first type Hukuhara derivative (classical Hukuhara derivative). Author studied the local and global existence and unique- 
ness results for (1.1) by using the method of successive approximations and the contraction principle. Malinowski [23] studied the existence and uniqueness result of a solution to the delay set-valued differential equation under condition that the right-hand side of the equation is Lipschitzian in the functional variable. In this paper, under the generalized Lipschitz condition, we obtain the local existence and uniqueness of two solutions to (1.1). Besides, we establish the global existence and uniqueness of two solutions to (1.1) by using some dissipative conditions [11]. We were inspired and motivated by the results of Stefanini and Bede [7, 24], Malinowski [23] and Lupulescu [10, 11]. The paper is organized as follows. In Section 2, we collect the fundamental notions and facts about a fuzzy set space, fuzzy differentiation. In Section 3, we discuss the FFDE with the generalized Hukuhara derivative. Under suitable conditions we prove the existence and uniqueness of the solution to FFDE by using two different methods. In Section 4, the existence and uniqueness of the sheaf solutions to sheaf fuzzy functional differential equations are studied. Finally, we give some examples to illustrate these results.

\section{Preliminaries and notation}

In this section, we give some notations and properties related to a fuzzy set space and summarize the major results for differentiation of fuzzy-set-valued mappings. Let $K_{c}\left(\mathbb{R}^{d}\right)$ denote the collection of all nonempty compact and convex subsets of $\mathbb{R}^{d}$ and scalar multiplication in $K_{c}\left(\mathbb{R}^{d}\right)$ as usual, i.e., for $A, B \in K_{c}\left(\mathbb{R}^{d}\right)$ and $\lambda \in \mathbb{R}$,

$$
A+B=\{a+b \mid a \in A, b \in B\}, \quad \lambda A=\{\lambda a \mid a \in A\} .
$$

The Hausdorff distance $d_{H}$ in $K_{c}\left(\mathbb{R}^{d}\right)$ is defined as follows:

$$
d_{H}(A, B)=\max \left\{\sup _{a \in A} \inf _{b \in B}\|a-b\|_{R^{n}}, \sup _{b \in B} \inf _{a \in A}\|a-b\|_{R^{n}}\right\},
$$

where $A, B \in K_{c}\left(\mathbb{R}^{d}\right),\|\cdot\|_{R^{n}}$ denotes the Euclidean norm in $\mathbb{R}^{d}$. It is known that $\left(K_{c}\left(\mathbb{R}^{d}\right), d_{H}\right)$ is complete, separable and locally compact. Define $E^{d}=\left\{\omega: \mathbb{R}^{d} \rightarrow[0,1]\right.$ such that $\omega(z)$ satisfies (i)-(iv) stated below\}

(i) $\omega$ is normal, that is, there exists $z_{0} \in \mathbb{R}^{d}$ such that $\omega\left(z_{0}\right)=1$;

(ii) $\omega$ is fuzzy convex, that is, for $0 \leq \lambda \leq 1$,

$$
\omega\left(\lambda z_{1}+(1-\lambda) z_{2}\right) \geq \min \left\{\omega\left(z_{1}\right), \omega\left(z_{2}\right)\right\}
$$

for any $z_{1}, z_{2} \in \mathbb{R}^{d}$;

(iii) $\omega$ is upper semicontinuous;

(iv) $[\omega]^{0}=\operatorname{cl}\left\{z \in \mathbb{R}^{d}: \omega(z)>0\right\}$ is compact, where cl denotes the closure in $\left(\mathbb{R}^{d},\|\cdot\|\right)$.

Although elements of $E^{d}$ are often called the fuzzy numbers, we shall just call them the fuzzy sets. For $\alpha \in(0,1]$, define $[\omega]^{\alpha}=\left\{z \in \mathbb{R}^{d} \mid \omega(z) \geq \alpha\right\}$. We will call this set an $\alpha$-cut ( $\alpha$-level set) of the fuzzy set $\omega$. For $\omega \in E^{d}$, one has that $[\omega]^{\alpha} \in K_{c}\left(\mathbb{R}^{d}\right)$ for every $\alpha \in[0,1]$. In the case $d=1$, the $\alpha$-cut set of a fuzzy number is a closed bounded interval $[\underline{\omega}(z, \alpha), \bar{\omega}(z, \alpha)]$, where $\underline{\omega}(z, \alpha)$ denotes the left-hand endpoint of $[\omega(z)]^{\alpha}$ and $\bar{\omega}(z, \alpha)$ the right-hand endpoint of $[\omega(z)]^{\alpha}$. It should be noted that for $a \leq b \leq c, a, b, c \in \mathbb{R}$, a triangular fuzzy number $\omega=(a, b, c)$ is given such that $\underline{\omega}(\alpha)=b-(1-\alpha)(b-a)$ and $\bar{\omega}(\alpha)=b+(1-\alpha)(c-b)$ are the endpoints of the $\alpha$-cut for all $\alpha \in[0,1]$. 
Let us denote by

$$
D_{0}\left[\omega_{1}, \omega_{2}\right]=\sup \left\{d_{H}\left(\left[\omega_{1}\right]^{\alpha},\left[\omega_{2}\right]^{\alpha}\right): 0 \leq \alpha \leq 1\right\}
$$

the distance between $\omega_{1}$ and $\omega_{2}$ in $E^{d}$, where $d_{H}\left(\left[\omega_{1}\right]^{\alpha},\left[\omega_{2}\right]^{\alpha}\right)$ is a Hausdorff distance between two sets $\left[\omega_{1}\right]^{\alpha},\left[\omega_{2}\right]^{\alpha}$ of $K_{c}\left(\mathbb{R}^{d}\right)$. Then $\left(E^{d}, D_{0}\right)$ is a complete space. Some properties of metric $D_{0}$ are as follows:

$$
\begin{aligned}
& D_{0}\left[\omega_{1}+\omega_{3}, \omega_{2}+\omega_{3}\right]=D_{0}\left[\omega_{1}, \omega_{2}\right], \\
& D_{0}\left[\lambda \omega_{1}, \lambda \omega_{2}\right]=|\lambda| D_{0}\left[\omega_{1}, \omega_{2}\right], \\
& D_{0}\left[\omega_{1}, \omega_{2}\right] \leq D_{0}\left[\omega_{1}, \omega_{3}\right]+D_{0}\left[\omega_{3}, \omega_{2}\right],
\end{aligned}
$$

for all $\omega_{1}, \omega_{2}, \omega_{3} \in E^{d}$ and $\lambda \in \mathbb{R}$. Let $\omega_{1}, \omega_{2} \in E^{d}$. If there exists $\omega_{3} \in E^{d}$ such that $\omega_{1}=$ $\omega_{2}+\omega_{3}$, then $\omega_{3}$ is called the $\mathrm{H}$-difference of $\omega_{1}, \omega_{2}$ and it is denoted by $\omega_{1} \ominus \omega_{2}$. Let us remark that $\omega_{1} \ominus \omega_{2} \neq \omega_{1}+(-1) \omega_{2}$.

Remark 2.1 ([13, 14]) If for fuzzy numbers $\omega_{1}, \omega_{2}, \omega_{3} \in E^{d}$ there exist Hukuhara differences $\omega_{1} \ominus \omega_{2}, \omega_{1} \ominus \omega_{3}$, then $D_{0}\left[\omega_{1} \ominus \omega_{2}, \hat{0}\right]=D_{0}\left[\omega_{1}, \omega_{2}\right]$ and $D_{0}\left[\omega_{1} \ominus \omega_{2}, \omega_{1} \ominus \omega_{3}\right]=$ $D_{0}\left[\omega_{2}, \omega_{3}\right]$.

Definition 2.1 ([25]) The generalized Hukuhara difference of two fuzzy numbers $u_{1}, u_{2} \in$ $E^{d}$ (gH-difference for short) is defined as follows:

$$
u_{1} \ominus_{g H} u_{2}=u_{3} \Leftrightarrow\left\{\begin{aligned}
\text { (i) } & u_{1}=u_{2}+u_{3}, \text { or } \\
\text { (ii) } & u_{2}=u_{1}+(-1) u_{3} .
\end{aligned}\right.
$$

The generalized Hukuhara differentiability was introduced in [24].

Definition 2.2 Let $t \in(a, b)$ and $h$ be such that $t+h \in(a, b)$, then the generalized Hukuhara derivative of a fuzzy-valued function $x:(a, b) \rightarrow E^{d}$ at $t$ is defined as

$$
D_{H}^{g} x(t)=\lim _{h \rightarrow 0} \frac{x(t+h) \ominus_{g H} x(t)}{h} .
$$

If $D_{H}^{g} x(t) \in E^{d}$ satisfying (2.1) exists, we say that $x$ is generalized Hukuhara differentiable (gH-differentiable for short) at $t$.

Theorem 2.1 ([25]) Let $x:[a, b] \rightarrow E^{1}, t \in(a, b)$ and put $[x(t)]^{\alpha}=[\underline{x}(t, \alpha), \bar{x}(t, \alpha)]$ for each $\alpha \in[0,1]$. The function $x$ is $g H$-differentiable if and only if $\underline{x}(t, \alpha)$ and $\bar{x}(t, \alpha)$ are differentiable with respect to $t$ for all $\alpha \in[0,1]$, and

$$
\left[D_{H}^{g} x(t)\right]^{\alpha}=\left[\min \left\{\underline{x}^{\prime}(t, \alpha), \bar{x}^{\prime}(t, \alpha)\right\}, \max \left\{\underline{x}^{\prime}(t, \alpha), \bar{x}^{\prime}(t, \alpha)\right\}\right] .
$$

Definition 2.3 Let $x:[a, b] \rightarrow E^{1}, t \in(a, b)$ and put $[x(t)]^{\alpha}=[\underline{x}(t, \alpha), \bar{x}(t, \alpha)]$ for each $\alpha \in$ $[0,1]$. We say that 
(i) $x$ is [(i)-gH]-differentiable at $t$ if $\underline{x}(t, \alpha), \bar{x}(t, \alpha)$ are differentiable functions and we have

$$
\left[D_{H}^{g} x(t)\right]^{\alpha}=\left[\underline{x}^{\prime}(t, \alpha), \bar{x}^{\prime}(t, \alpha)\right] .
$$

(ii) $x$ is [(ii)-gH]-differentiable at $t$ if $\underline{x}(t, \alpha), \bar{x}(t, \alpha)$ are differentiable functions and we have

$$
\left[D_{H}^{g} x(t)\right]^{\alpha}=\left[\bar{x}^{\prime}(t, \alpha), \underline{x}^{\prime}(t, \alpha)\right]
$$

Definition 2.4 ([25]) We say that a point $t \in(a, b)$ is a switching point for the differentiability of $x$, if in any neighborhood $V$ of $t$ there exist points $t_{1}<t<t_{2}$ such that

(type I) at $t_{1}$ (2.2) holds while (2.3) does not hold and at $t_{2}$ (2.3) holds and (2.2) does not hold, or

(type II) at $t_{1}$ (2.3) holds while (2.2) does not hold and at $t_{2}$ (2.2) holds and (2.3) does not hold.

Lemma 2.1 (Bede and Gal [7]) If $x(t)=\left(z_{1}(t), z_{2}(t), z_{3}(t)\right)$ is a triangular fuzzy-valued function, then

(i) if $x$ is $[(i)$-gH]-differentiable (i.e., Hukuhara differentiable), then $D_{H}^{g} x(t)=\left(z_{1}^{\prime}(t), z_{2}^{\prime}(t), z_{3}^{\prime}(t)\right)$

(ii) if $x$ is $\left[(i i)\right.$-gH]-differentiable, then $D_{H}^{g} x(t)=\left(z_{3}^{\prime}(t), z_{2}^{\prime}(t), z_{1}^{\prime}(t)\right)$.

Lemma 2.2 (see [8]) Let $x \in E^{1}$ and put $[x(t)]^{\alpha}=[\underline{x}(t, \alpha), \bar{x}(t, \alpha)]$ for each $\alpha \in[0,1]$.

(i) If $x$ is $[(i)$-gH]-differentiable, then $\underline{x}(t, \alpha), \bar{x}(t, \alpha)$ are differentiable functions and $\left[D_{H}^{g} x(t)\right]^{\alpha}=\left[\underline{x}^{\prime}(t, \alpha), \bar{x}^{\prime}(t, \alpha)\right]$

(ii) If $x$ is $[(i i)$-gH]-differentiable, then $\underline{x}(t, \alpha), \bar{x}(t, \alpha)$ are differentiable functions and we have $\left[D_{H}^{g} x(t)\right]^{\alpha}=\left[\bar{x}^{\prime}(t, \alpha), \underline{x}^{\prime}(t, \alpha)\right]$.

In the following, we shall recall some concepts which were introduced and studied in [10]. In [10], the author introduced a new concept of inner product on the fuzzy space $E^{d}$.

For $x, y \in E^{d}$, we consider the function $\psi(\cdot ; x, y): \mathbb{R}^{+} \rightarrow \mathbb{R}$, defined by

$$
\psi(h ; x, y)=\frac{D_{0}[x+h y, \hat{0}]-D_{0}[x, \hat{0}]}{h} .
$$

Lemma 2.3 (see [10]) For every $x, y \in E^{d}$, there exists the limit

$$
[x, y]_{+}=\lim _{h \rightarrow 0^{+}} \frac{D_{0}[x+h y, \hat{0}]-D_{0}[x, \hat{0}]}{h} .
$$

Definition 2.5 (see [10]) For any $x, y \in E^{d}$, we define the inner product on $E^{d}$ by

$$
(x, y)_{+}=\lim _{h \rightarrow 0^{+}} \frac{D_{0}^{2}[x+h y, \hat{0}]-D_{0}^{2}[x, \hat{0}]}{2 h} .
$$

Corollary 2.1 (see [10]) For every $x, y \in E^{d}$, we have that

$$
(x, y)_{+}=D_{0}[x, \hat{0}][x, y]_{+} .
$$


Theorem 2.2 (see [10]) If $x(\cdot):[a, b] \rightarrow E^{d}$ is continuously differentiable on $[a, b]$, then

$$
\frac{1}{2} \frac{d^{+}}{d t} D_{0}^{2}[x(t), \hat{0}]=\left(x(t), D_{H}^{g} x(t)\right)_{+}
$$

for every $t \in[a, b]$.

By help of these concepts we formulate some dissipative conditions for FFDEs (1.1) and, under these conditions, we establish the global existence and uniqueness of a solution of functional fuzzy differential equations.

\section{Fuzzy functional differential equations}

For a positive number $\sigma$, we denote by $C_{\sigma}$ the space $C\left([-\sigma, 0], E^{d}\right)$, we denote by

$$
D_{\sigma}[x, y]=\sup _{t \in[-\sigma, 0]} D_{0}[x(t), y(t)]
$$

the metric on the space $C_{\sigma}$. Define $I=\left[t_{0}, t_{0}+p\right], J=\left[t_{0}-\sigma, t_{0}\right] \cup I=\left[t_{0}-\sigma, t_{0}+p\right]$. Then, for each $t \in I$, we denote by $x_{t}$ the element of $C_{\sigma}$ defined by $x_{t}(s)=x(t+s), s \in[-\sigma, 0]$.

Let us consider the fuzzy functional differential equation with the generalized Hukuhara derivative of the form

$$
\begin{cases}D_{H}^{g} x(t)=f\left(t, x_{t}\right), & t \geq t_{0}, \\ x(t)=\varphi\left(t-t_{0}\right)=\varphi_{0}, & t_{0} \geq t \geq t_{0}-\sigma,\end{cases}
$$

where $f: I \times C_{\sigma} \rightarrow E^{d}, \varphi \in C_{\sigma}$, and the symbol $D_{H}^{g}$ denotes the generalized Hukuhara derivative from Definition 2.2. By a solution to equation (3.1) we mean a fuzzy mapping $x \in C\left(J, E^{d}\right)$ that satisfies: $x(t)=\varphi\left(t-t_{0}\right)$ for $t \in\left[t_{0}-\sigma, t_{0}\right], x$ is differentiable on $\left[t_{0}, t_{0}+p\right]$ and $D_{H}^{g} x(t)=f\left(t, x_{t}\right)$ for $t \in I$.

Lemma 3.1 (see [10]) Assume that $f \in C\left(I \times C_{\sigma}, E^{d}\right)$ and $x \in C\left(J, E^{d}\right)$. Then the fuzzy mapping $t \rightarrow f\left(t, x_{t}\right)$ belongs to $C\left(I, E^{d}\right)$.

Remark 3.1 (see [10]) Under assumptions of the lemma above we have that the mapping $t \rightarrow f\left(t, x_{t}\right)$ is integrable over the interval $I$.

Remark 3.2 (see [10]) If $f: I \times C_{\sigma} \rightarrow E^{d}$ is a jointly continuous function and $x \in C\left(J, E^{d}\right)$, then the mapping $t \rightarrow f\left(t, x_{t}\right)$ is bounded on each compact interval $I$. Also, the function $t \rightarrow f(t, \hat{0})$ is bounded on $I$.

The following lemma is similar to the result proved in $[10,23,26]$.

Lemma 3.2 The fuzzy functional differential equation (3.1) is equivalent to the following integral equation:

$$
\begin{cases}x(t)=\varphi\left(t-t_{0}\right) & \text { for } t \in\left[t_{0}-\sigma, t_{0}\right] \\ x(t) \ominus_{g H} \varphi(0)=\int_{t_{0}}^{t} f\left(s, x_{s}\right) d s, & t \in I .\end{cases}
$$


Two cases of existence of the generalized $\mathrm{H}$-difference imply that the integral equation in Lemma 3.2 is actually a unified formulation for one of the integral equations

$$
\begin{cases}x(t)=\varphi\left(t-t_{0}\right) & \text { for } t \in\left[t_{0}-\sigma, t_{0}\right], \\ x(t) \ominus \varphi(0)=\int_{t_{0}}^{t} f\left(s, x_{s}\right) d s, & t \in I,\end{cases}
$$

and

$$
\begin{cases}x(t)=\varphi\left(t-t_{0}\right) & \text { for } t \in\left[t_{0}-\sigma, t_{0}\right] \\ \varphi(0) \ominus x(t)=(-1) \int_{t_{0}}^{t} f\left(s, x_{s}\right) d s, & t \in I,\end{cases}
$$

with $\ominus$ being the classical Hukuhara difference. Now, we consider $\widehat{x}$ and $\widetilde{x}$ to be the solutions of equation (3.1) in [(i)-gH]-differentiability type and [(ii)-gH]-differentiability type, respectively, then by using Lemma 3.2 we have

$$
\begin{aligned}
& \begin{cases}\widehat{x}(t)=\varphi\left(t-t_{0}\right) & \text { for } t \in\left[t_{0}-\sigma, t_{0}\right], \\
\widehat{x}(t)=\varphi(0)+\int_{t_{0}}^{t} f\left(s, \widehat{x}_{s}\right) d s, & t \in I,\end{cases} \\
& \begin{cases}\widetilde{x}(t)=\varphi\left(t-t_{0}\right) & \text { for } t \in\left[t_{0}-\sigma, t_{0}\right], \\
\widetilde{x}(t)=\varphi(0) \ominus(-1) \int_{t_{0}}^{t} f\left(s, \widetilde{x}_{s}\right) d s, & t \in I .\end{cases}
\end{aligned}
$$

Definition 3.1 Let $x: J \rightarrow E^{d}$ be a fuzzy function which is [(i)-gH]-differentiable. If $x$ and its derivative satisfy problem (3.1), we say that $x$ is a (i)-solution of problem (3.1).

Definition 3.2 Let $x: J \rightarrow E^{d}$ be a fuzzy function which is [(ii)-gH]-differentiable. If $x$ and its derivative satisfy problem (3.1), we say that $x$ is a (ii)-solution of problem (3.1).

\subsection{Local existence}

The following comparison principle is fundamental in investigation of the global existence of solutions of functional fuzzy differential equations.

Theorem 3.1 ([27]) Let $m \in C\left(\left[t_{0}-\sigma, \infty\right), \mathbb{R}\right)$ and satisfy the inequality

$$
D^{+} m(t) \leq g\left(t,\left|m_{t}\right|_{\sigma}\right), \quad t>t_{0},
$$

where $g \in C\left(\left[t_{0}, \infty\right) \times \mathbb{R}^{+}, \mathbb{R}^{+}\right)$. Assume that $r(t)=r\left(t, t_{0}, u_{0}\right)$ is the maximal solution of the IVP

$$
\frac{d}{d t} u=g(t, u), \quad u\left(t_{0}\right)=u_{0} \geq 0
$$

existing on $\left[t_{0}, \infty\right)$. Then, if $\left|m_{t_{0}}\right|_{\sigma} \leq u_{0}$, we have $m(t) \leq r(t), t \in\left[t_{0}, \infty\right)$.

Let $\rho>0$ be a given constant, and let $\Omega\left(x^{0}, \rho\right)=\left\{x \in E^{d}: D_{0}\left[x, x^{0}\right] \leq \rho\right\}$ and $S\left(x^{0}, \rho\right)=$ $\left\{\xi \in C_{\sigma}: D_{\sigma}\left[\xi, x^{0}\right] \leq \rho\right\}$. Let us consider the mappings $f: I \times S\left(x^{0}, \rho\right) \rightarrow E^{d}$ and $g: I \times$ $[0, \rho] \rightarrow \mathbb{R}^{+}$, where

$$
x^{0}(t)= \begin{cases}\varphi\left(t-t_{0}\right), & t \in\left[t_{0}-\sigma, t_{0}\right] \\ \varphi(0), & t \in I\end{cases}
$$


Under the generalized Lipschitz condition we obtain the existence and uniqueness of two solutions to FFDE. To prove this assertion, we use an idea of successive approximations.

Theorem 3.2 Assume that

(i) $f \in C\left(I \times S\left(x^{0}, \rho\right), E^{d}\right)$ and $D_{0}[f(t, \xi), \hat{0}] \leq M_{1}, \forall(t, \xi) \in I \times S\left(x^{0}, \rho\right)$;

(ii) $g \in C\left(I \times[0, \rho], \mathbb{R}^{+}\right), g(t, 0) \equiv 0$ and $0 \leq g(t, u) \leq M_{2}, \forall t \in I, 0 \leq u \leq \rho$ such that $g(t, u)$ is nondecreasing on $u$, the IVP

$$
\frac{d u}{d t}=g(t, u(t)), \quad u\left(t_{0}\right)=0
$$

has only the solution $u(t) \equiv 0$ on $I$;

(iii) $D_{0}[f(t, \xi), f(t, \psi)] \leq g\left(t, D_{\sigma}[\xi, \psi]\right), \forall(t, \xi),(t, \psi) \in I \times S\left(x^{0}, \rho\right)$, and $D_{\sigma}[\xi, \psi] \leq M_{3}$.

Then the following successive approximations given by

$$
\begin{aligned}
& \widehat{x}^{0}(t)=\left\{\begin{array}{ll}
\varphi\left(t-t_{0}\right), & t \in\left[t_{0}-\sigma, t_{0}\right], \\
\varphi(0), & t \in I,
\end{array} \text { and for } n=0,1,2, \ldots,\right. \\
& \widehat{x}^{n+1}(t)= \begin{cases}\varphi\left(t-t_{0}\right), & t \in\left[t_{0}-\sigma, t_{0}\right], \\
\varphi(0)+\int_{t_{0}}^{t} f\left(s, \widehat{x}_{s}^{n}\right) d s, & t \in\left[t_{0}, t_{0}+p\right],\end{cases}
\end{aligned}
$$

for the case of $[(i)-g H]$-differentiability, and

$$
\begin{aligned}
& \widetilde{x}^{0}(t)= \begin{cases}\varphi\left(t-t_{0}\right), & t \in\left[t_{0}-\sigma, t_{0}\right], \\
\varphi(0), & t \in I,\end{cases} \\
& \widetilde{x}^{n+1}(t)= \begin{cases}\varphi\left(t-t_{0}\right), & t \in\left[t_{0}-\sigma, t_{0}\right], \\
\varphi(0) \ominus(-1) \int_{t_{0}}^{t} f\left(s, \widetilde{x}_{s}^{n}\right) d s, & t \in\left[t_{0}, t_{0}+d\right],\end{cases}
\end{aligned}
$$

for the case of $[(i i)$-gH]-differentiability (where $d>0$ such that the sequence (3.9) is well defined, i.e., the foregoing Hukuhara differences do exist), converge uniformly to two unique solutions $\widehat{x}(t)$ and $\widetilde{x}(t)$ of $(3.1)$, respectively, on $\left[t_{0}, t_{0}+r\right]$ where $r=\min \left\{p, \rho / M_{1}, \rho / M_{2}, d\right\}$.

Proof We prove that for the case of [(ii)-gH]-differentiability, the proof of the other case is similar. For $t_{0} \leq t_{1} \leq t_{2} \leq t_{0}+r, n=1,2, \ldots$ and $M=\max \left\{M_{1}, M_{2}\right\}$, we have

$$
\begin{aligned}
D_{0}\left[\widetilde{x}^{n}\left(t_{1}\right), \widetilde{x}^{n}\left(t_{2}\right)\right] & =D_{0}\left[\varphi(0) \ominus(-1) \int_{t_{0}}^{t_{1}} f\left(s, \widetilde{x}_{s}^{n-1}\right) d s, \varphi(0) \ominus(-1) \int_{t_{0}}^{t_{2}} f\left(s, \widetilde{x}_{s}^{n-1}\right) d s\right] \\
& \leq \int_{t_{1}}^{t_{2}} D_{0}\left[f\left(s, \widetilde{x}_{s}^{n-1}\right), \hat{0}\right] d s \leq M\left(t_{2}-t_{1}\right)<\varepsilon
\end{aligned}
$$

provided $t_{2}-t_{1}<\delta$, where $\delta=\frac{\varepsilon}{M}$, proving that $\widetilde{x}^{n}(t)$ is continuous on $\left[t_{0}, t_{0}+r\right]$.

Similarly,

$$
\begin{aligned}
D_{0}\left[\widetilde{x}^{1}(t), \widetilde{x}^{0}(t)\right] & =D_{0}\left[\varphi(0) \ominus(-1) \int_{t_{0}}^{t} f\left(s, \widetilde{x}_{s}^{0}\right) d s, \varphi(0)\right] \\
& \leq \int_{t_{0}}^{t} D_{0}\left[f\left(s, \widetilde{x}_{s}^{0}\right), \hat{0}\right] d s \leq \rho .
\end{aligned}
$$


Thus, it is easily obtain that the successive approximations are continuous and satisfy the following relation:

$$
D_{0}\left[\widetilde{x}^{n+1}(t), \widetilde{x}^{0}(t)\right] \leq \rho, \quad \forall t \in\left[t_{0}, t_{0}+r\right], n=0,1,2,3, \ldots
$$

Hence, $\widetilde{x}^{n+1} \in C\left(\left[t_{0}, t_{0}+r\right], \Omega\left(x^{0}, \rho\right)\right)$. Observe also that the Hukuhara differences $\widetilde{x}^{n+1}(t-$ h) $\ominus \widetilde{x}^{n+1}(t), \widetilde{x}^{n+1}(t) \ominus \widetilde{x}^{n+1}(t+h)$ exist and

$$
\begin{aligned}
& \widetilde{x}^{n+1}(t-h) \ominus \widetilde{x}^{n+1}(t)=(-1) \int_{t-h}^{t} f\left(s, \widetilde{x}^{n}(s)\right) d s, \\
& \widetilde{x}^{n+1}(t) \ominus \widetilde{x}^{n+1}(t+h)=(-1) \int_{t}^{t+h} f\left(s, \widetilde{x}^{n}(s)\right) d s .
\end{aligned}
$$

Hence we infer that $D_{H}^{g} \widetilde{x}^{n+1}(t)=f\left(t, \widetilde{x}_{t}^{n}\right), \widetilde{x}^{n+1}\left(t_{0}\right)=\widetilde{x}^{0}$ for $t \in\left[t_{0}, t_{0}+r\right](n=0,1,2, \ldots)$.

Now, we define the following successive approximations of (3.7) for $r=\min \{p, \rho / M\}$ :

$$
u^{0}(t)=M\left(t-t_{0}\right), \quad u^{n+1}(t)=\int_{t_{0}}^{t} g\left(s, u^{n}(s)\right) d s, \quad t \in\left[t_{0}, t_{0}+r\right](n=0,1,2, \ldots) .
$$

Then we get immediately

$$
u^{1}(t)=\int_{t_{0}}^{t} g\left(s, u^{0}(s)\right) d s \leq M_{2}\left(t-t_{0}\right) \leq u^{0}(t) \leq \rho, \quad \forall t \in\left[t_{0}, t_{0}+r\right] .
$$

Hence, by the inductive method and in view that $g(t, u)$ is nondecreasing on $u$, we get

$$
0 \leq u^{n+1}(t) \leq u^{n}(t) \leq \rho, \quad \forall t \in\left[t_{0}, t_{0}+r\right], n=0,1,2,3, \ldots
$$

As $\left|\frac{d u^{n+1}(t)}{d t}\right|=\left|g\left(t, u^{n}(t)\right)\right| \leq M_{2}$, the sequence $\left\{u^{n}\right\}$ is equicontinuous. Hence, we can conclude by the Ascoli-Arzela theorem and the monotonicity of the sequence $\left\{u^{n}(t)\right\}$ that $\lim _{n \rightarrow \infty} u^{n}(t)=u(t)$ uniformly on $\left[t_{0}, t_{0}+r\right]$ and $u(t)=\int_{t_{0}}^{t} g(s, u(s)) d s$. Thus, $u \in C\left(\left[t_{0}, t_{0}+\right.\right.$ $r],[0, \rho])$ and $u(t)$ is the solution of initial value problem (3.7). From assumption (ii), we get $u(t) \equiv 0$. In addition, we have

$$
D_{0}\left[\widetilde{x}^{1}(t), \widetilde{x}^{0}(t)\right] \leq \int_{t_{0}}^{t} D_{0}\left[f\left(s, \widetilde{x}_{s}^{0}\right), \hat{0}\right] d s \leq M_{1}\left(t-t_{0}\right) \leq u^{0}(t)
$$

and

$$
\begin{aligned}
\sup _{\varsigma \in[-\sigma, 0]} D_{0}\left[\widetilde{x}^{1}(t+\varsigma), \widetilde{x}^{0}(t+\varsigma)\right] & \leq \sup _{\varsigma \in[-\sigma, 0]} \int_{t_{0}}^{t+\varsigma} D_{0}\left[f\left(s, \widetilde{x}_{s}^{0}\right), \hat{0}\right] d s \\
& \leq \sup _{\theta \in[t-\sigma, t]} \int_{t_{0}}^{\theta} D_{0}\left[f\left(s, \widetilde{x}_{s}^{0}\right), \hat{0}\right] d s \\
& \leq M_{1} \sup _{\theta \in[t-\sigma, t]}\left(\theta-t_{0}\right) \leq u^{0}(t) .
\end{aligned}
$$


Suppose $D_{0}\left[x^{i}(t), x^{i-1}(t)\right] \leq u^{i-1}(t)$ and $\sup _{\varsigma \in[-\sigma, 0]} D_{0}\left[x^{i}(t+\varsigma), x^{i-1}(t+\varsigma)\right] \leq u^{i-1}(t)$, then by assumption (iii), we get

$$
\begin{aligned}
D_{0}\left[\widetilde{x}^{i+1}(t), \widetilde{x}^{i}(t)\right] & \leq \int_{t_{0}}^{t} D_{0}\left[f\left(s, \widetilde{x}_{s}^{i}\right), f\left(s, \widetilde{x}_{s}^{i-1}\right)\right] d s \\
& \leq \int_{t_{0}}^{t} g\left(s, \sup _{s \in[-\sigma, 0]} D_{0}\left[\widetilde{x}^{i}(s+\varsigma), \widetilde{x}^{i-1}(s+\varsigma)\right]\right) d s \\
& \leq \int_{t_{0}}^{t} g\left(s, u^{i-1}(s)\right) d s=u^{i}(t) .
\end{aligned}
$$

Thus, by mathematical induction, we obtain: $D_{0}\left[\widetilde{x}^{n+1}(t), \widetilde{x}^{n}(t)\right] \leq u^{n}(t), \forall t \in\left[t_{0}, t_{0}+r\right], n=$ $0,1,2, \ldots$ Applying this property we have, for $t \in\left[t_{0}, t_{0}+r\right]$ and for $n=0,1,2, \ldots$,

$$
D_{0}\left[D_{H}^{g} \widetilde{x}^{n+1}(t), D_{H}^{g} \widetilde{x}^{n}(t)\right] \leq D_{0}\left[f\left(t, \widetilde{x}_{t}^{n}\right), f\left(t, \widetilde{x}_{t}^{n-1}\right)\right] \leq g\left(t, D_{\sigma}\left[\widetilde{x}_{t}^{n}, \widetilde{x}_{t}^{n-1}\right]\right) \leq g\left(t, u^{n-1}(t)\right) .
$$

Assume $m \geq n$, then one can easily obtain

$$
\begin{aligned}
D_{0}\left[D_{H}^{g} \widetilde{x}^{n}(t), D_{H}^{g} \widetilde{x}^{m}(t)\right] \leq & D_{0}\left[D_{H}^{g} \widetilde{x}^{n}(t), D_{H}^{g} \widetilde{x}^{n+1}(t)\right]+D_{0}\left[D_{H}^{g} \widetilde{x}^{n+1}(t), D_{H}^{g} \widetilde{x}^{m+1}(t)\right] \\
& +D_{0}\left[D_{H}^{g} \widetilde{x}^{m+1}(t), D_{H}^{g} \widetilde{x}^{m}(t)\right] \\
\leq & 2 g\left(t, u^{n-1}(t)\right)+g\left(t, D_{\sigma}\left[\widetilde{x}^{n}(t), \widetilde{x}^{m}(t)\right]\right) .
\end{aligned}
$$

Further, we have, for small positive $h$,

$$
\begin{aligned}
D_{0} & {\left[\widetilde{x}^{n}(t+h), \widetilde{x}^{m}(t+h)\right] } \\
& =D_{0}\left[\varphi(0) \ominus(-1) \int_{t_{0}}^{t+h} f\left(s, \widetilde{x}_{s}^{n-1}\right) d s, \varphi(0) \ominus(-1) \int_{t_{0}}^{t+h} f\left(s, \widetilde{x}_{s}^{m-1}\right) d s\right] \\
& =D_{0}\left[\widetilde{x}^{n}(t) \ominus(-1) \int_{t}^{t+h} f\left(s, \widetilde{x}_{s}^{n-1}\right) d s, \widetilde{x}^{m}(t) \ominus(-1) \int_{t}^{t+h} f\left(s, \widetilde{x}_{s}^{m-1}\right) d s\right] \\
& \leq D_{0}\left[\widetilde{x}^{n}(t), \widetilde{x}^{m}(t)\right]+D_{0}\left[\widetilde{x}^{n}(t) \ominus \widetilde{x}^{n}(t+h), \widetilde{x}^{m}(t) \ominus \widetilde{x}^{m}(t+h)\right] .
\end{aligned}
$$

Therefore, we obtain the Dini derivative $D^{+}$of the function $D_{0}\left[\widetilde{x}^{n}(t), \widetilde{x}^{m}(t)\right]$ as follows:

$$
\begin{aligned}
D^{+} D_{0}\left[\widetilde{x}^{n}(t), \widetilde{x}^{m}(t)\right] & =\varlimsup_{h \rightarrow 0^{+}} \frac{1}{h}\left[D_{0}\left[\widetilde{x}^{n}(t+h), \widetilde{x}^{m}(t+h)\right]-D_{0}\left[\widetilde{x}^{n}(t), \widetilde{x}^{m}(t)\right]\right] \\
& \leq \varlimsup_{h \rightarrow 0^{+}} \frac{1}{h} D_{0}\left[\widetilde{x}^{n}(t) \ominus \widetilde{x}^{n}(t+h), \widetilde{x}^{m}(t) \ominus \widetilde{x}^{m}(t+h)\right] \\
& \leq \varlimsup_{h \rightarrow 0^{+}} D_{0}\left[\left(-\frac{1}{h}\right)\left(\widetilde{x}^{n}(t) \ominus \widetilde{x}^{n}(t+h)\right),\left(-\frac{1}{h}\right)\left(\widetilde{x}^{m}(t) \ominus \widetilde{x}^{m}(t+h)\right)\right] \\
& =D_{0}\left[D_{H}^{g} \widetilde{x}^{n}(t), D_{H}^{g} \widetilde{x}^{m}(t)\right] \leq 2 g\left(t, u^{n-1}(t)\right)+g\left(t, D_{\sigma}\left[\widetilde{x}^{n}(t), \widetilde{x}^{m}(t)\right]\right) .
\end{aligned}
$$

Since $g\left(t, u^{n-1}(t)\right)$ uniformly converges to 0 , then for arbitrary $\varepsilon>0$, there exists a natural number $n_{0}$ such that

$$
D^{+} D_{0}\left[\widetilde{x}^{n}(t), \widetilde{x}^{m}(t)\right] \leq g\left(t, D_{\sigma}\left[\widetilde{x}^{n}(t), \widetilde{x}^{m}(t)\right]\right)+\varepsilon \quad \text { for } m \geq n \geq n_{0}
$$


From the fact that $D_{0}\left[\widetilde{x}^{n}\left(t_{0}\right), \widetilde{x}^{m}\left(t_{0}\right)\right]=0<\varepsilon$ and by using Theorem 3.1, we have

$$
D_{0}\left[\widetilde{x}^{n}(t), \widetilde{x}^{m}(t)\right] \leq u_{\varepsilon}(t), \quad t \in\left[t_{0}, t_{0}+r\right], m \geq n \geq n_{0},
$$

where $u_{\varepsilon}(t)$ is the maximal solution to the following IVP for each $n$ :

$$
\frac{d u_{\varepsilon}(t)}{d t}=g\left(t, u_{\varepsilon}(t)\right)+\varepsilon .
$$

Due to Proposition 2.2 in [5] one can infer that $\left\{u_{\varepsilon}(\cdot)\right\}$ converges uniformly to the maximal solution $u(t) \equiv 0$ of (3.7) on $\left[t_{0}, t_{0}+r\right]$ as $\varepsilon \rightarrow 0$. Hence, by virtue of (3.9), we infer that $\left\{\widetilde{x}^{n}\right\}$ converges uniformly to a continuous function $\tilde{x}:\left[t_{0}, t_{0}+r\right] \rightarrow \Omega\left(x_{0}, \rho\right)$. Note that $\tilde{x}$ is the desired solution to (3.1). Indeed, for every $t \in\left[t_{0}, t_{0}+r\right]$, we have

$$
\begin{aligned}
D_{0} & {\left[\varphi(0), \widetilde{x}(t)+(-1) \int_{t_{0}}^{t} f\left(s, \widetilde{x}_{s}\right) d s\right] } \\
& =D_{0}\left[\widetilde{x}^{n}(t)+(-1) \int_{t_{0}}^{t} f\left(s, x_{s}^{n-1}\right) d s, \widetilde{x}(t)+(-1) \int_{t_{0}}^{t} f\left(s, \widetilde{x}_{s}\right) d s\right] \\
& \leq D_{0}\left[\widetilde{x}^{n}(t), \widetilde{x}(t)\right]+\int_{t_{0}}^{t} D_{0}\left[f\left(s, \widetilde{x}_{s}^{n-1}\right), f\left(s, \widetilde{x}_{s}\right)\right] d s .
\end{aligned}
$$

The summands in the last expression converge to 0 . Due to Lemma 3.2 the function $\widetilde{x}$ is the (ii)-solution to (3.1).

Now, we show the uniqueness of (ii)-solution of equation (3.1). Suppose that $\tilde{y}(t)$ is another local (ii)-solution to (3.1) on the interval $\left[t_{0}, t_{0}+r\right]$. Define $m(t)=D_{0}\left[\widetilde{x}\left(t_{0}, \varphi_{0}\right)(t)\right.$, $\left.\tilde{y}\left(t_{0}, \varphi_{0}\right)(t)\right]$, then $m\left(t_{0}\right)=0$ and

$$
D^{+} m(t)=D_{0}\left[D_{H}^{g} \tilde{x}(t), D_{H}^{g} \tilde{y}(t)\right] \leq g\left(t, D_{\sigma}\left[\tilde{x}_{t}, \tilde{y}_{t}\right]\right) \leq g\left(t,\left|m_{t}\right|_{\sigma}\right) .
$$

Hence, by Theorem 3.1, we have $m(t) \leq u(t)$ for all $t \in\left[t_{0}, t_{0}+r\right]$, where $u(t) \equiv 0$ is the maximal solution of IVP (3.7). Therefore $\widetilde{x}(t) \equiv \tilde{y}(t)$, which completes the proof.

Corollary 3.1 Let $\varphi\left(t-t_{0}\right) \in C_{\sigma}$ and suppose that $f \in C\left(I \times S\left(x^{0}, \rho\right)\right.$, $\left.E^{d}\right)$ satisfies the condition: there exists a constant $L>0$ such that for every $\xi, \psi \in S\left(x^{0}, \rho\right)$ it holds

$$
D_{0}[f(t, \xi), f(t, \psi)] \leq L D_{\sigma}[\xi, \psi] .
$$

Moreover, there exists $M>0$ such that $D_{0}[f(t, \xi), \hat{0}] \leq M_{1}$.

Then the following successive approximations given by

$$
\begin{aligned}
& \widehat{x}^{0}(t)=\left\{\begin{array}{ll}
\varphi\left(t-t_{0}\right), & t \in\left[t_{0}-\sigma, t_{0}\right], \\
\varphi(0), & t \in I,
\end{array} \text { and for } n=0,1,2, \ldots,\right. \\
& \widehat{x}^{n+1}(t)= \begin{cases}\varphi\left(t-t_{0}\right), & t \in\left[t_{0}-\sigma, t_{0}\right], \\
\varphi(0)+\int_{t_{0}}^{t} f\left(s, \widehat{x}_{s}^{n}\right) d s, & t \in I,\end{cases}
\end{aligned}
$$


for the case of $[(i)-g H]$-differentiability, and

$$
\begin{aligned}
& \widetilde{x}^{0}(t)=\left\{\begin{array}{ll}
\varphi\left(t-t_{0}\right), & t \in\left[t_{0}-\sigma, t_{0}\right], \\
\varphi(0), & t \in I,
\end{array} \text { and for } n=0,1,2, \ldots,\right. \\
& \widetilde{x}^{n+1}(t)= \begin{cases}\varphi\left(t-t_{0}\right), \\
\varphi(0) \ominus(-1) \int_{t_{0}}^{t} f\left(s, \widetilde{x}_{s}^{n}\right) d s, & t \in I,\end{cases}
\end{aligned}
$$

for the case of $[(i i)$-gH]-differentiability, converge uniformly to two unique solutions $\widehat{x}(t)$ and $\widetilde{x}(t)$ of $(3.1)$, respectively, on $\left[t_{0}, t_{0}+r\right]$ where $r=\min \left\{p, \rho / M_{1}, \rho / M_{2}\right\}$.

Proof The proof is obtained immediately by taking in Theorem 3.2, $g(t, u)=L D_{\sigma}[\xi, \psi]$.

\subsection{Global existence}

Next, we shall establish the global existence and uniqueness results for FFDE (3.1). For the global existence and uniqueness, we use the dissipative conditions which were introduced and studied in [11]. We now prove a comparison theorem, which is a useful tool in proving the global existence theorem.

Theorem 3.3 Assume that $f \in C\left(\mathbb{R}^{+} \times C_{\sigma}, E^{d}\right)$ and $D_{0}[f(t, \xi), f(t, \psi)] \leq g\left(t, D_{\sigma}[\xi, \psi]\right)$ for $\xi, \psi \in C_{\sigma}, t \in \mathbb{R}^{+}$, where $g \in C\left(\mathbb{R}^{+} \times R^{+}, \mathbb{R}^{+}\right)$. Let $r\left(t, t_{0}, u_{0}\right)$ be the maximal solution of

$$
\left\{\begin{array}{l}
\frac{d u}{d t}=g(t, u), \\
u\left(t_{0}\right)=u_{0} \geq 0
\end{array}\right.
$$

for $t \geq t_{0}$. Then, if $\xi(t), \psi(t)$ are any (ii)-solutions of FFDE (3.1) such that $\xi_{0}, \psi_{0} \in C_{\sigma}$ exist for $t \in \mathbb{R}^{+}$, we have $D_{0}[\xi(t), \psi(t)] \leq r\left(t, t_{0}, u_{0}\right)$, provided that $D_{\sigma}\left[\xi_{0}, \psi_{0}\right] \leq u_{0}$.

Proof Since $\xi, \psi$ are solutions of FFDE (3.1) and [(ii)-gH]-differentiable, we have: for $h>0$ small enough, there exist the Hukuhara differences $\xi(t-h) \ominus \xi(t), \psi(t-h) \ominus \psi(t)$. Now, for $t \in \mathbb{R}^{+}$, set $m(t)=D_{0}[\xi(t), \psi(t)]$, we have

$$
\begin{aligned}
m(t-h)-m(t)= & D_{0}[\xi(t-h), \psi(t-h)]-D_{0}[\xi(t), \psi(t)] \\
\leq & D_{0}\left[\xi(t-h), \xi(t)+(-1) h f\left(t, \xi_{t}\right)\right] \\
& +D_{0}\left[\xi(t)+(-1) h f\left(t, \xi_{t}\right), \psi(t)+(-1) h f\left(t, \psi_{t}\right)\right] \\
& +D_{0}\left[\psi(t)+(-1) h f\left(t, \psi_{t}\right), \psi(t-h)\right]-D_{0}[\xi(t), \psi(t)] \\
\leq & D_{0}\left[\xi(t-h), \xi(t)+(-1) h f\left(t, \xi_{t}\right)\right]+D_{0}\left[\psi(t)+(-1) h f\left(t, \psi_{t}\right), \psi(t-h)\right] \\
& +h D_{0}\left[f\left(t, \xi_{t}\right), f\left(t, \psi_{t}\right)\right],
\end{aligned}
$$

from which we get

$$
\begin{aligned}
\frac{m(t-h)-m(t)}{h} \leq & D_{0}\left[\frac{\xi(t-h) \ominus \xi(t)}{-h}, f\left(t, \xi_{t}\right)\right]+D_{0}\left[f\left(t, \psi_{t}\right), \frac{\psi(t-h) \ominus \psi(t)}{-h}\right] \\
& +D_{0}\left[f\left(t, \xi_{t}\right), f\left(t, \psi_{t}\right)\right] .
\end{aligned}
$$


Taking $\liminf$ as $h \rightarrow 0^{+}$yields

$$
D^{-} m(t)=\liminf _{h \rightarrow 0^{+}} \frac{1}{h}[m(t-h)-m(t)] \leq g\left(t, D_{\sigma}\left[\xi_{t}, \psi_{t}\right]\right)=g\left(t, D_{\sigma}\left[\xi_{0}, \psi_{0}\right]\right),
$$

which together with the fact that $D_{\sigma}\left[\xi_{0}, \psi_{0}\right] \leq u_{0}$ and by Theorem 3.1 gives

$$
D_{0}[\xi(t), \psi(t)] \leq r\left(t, t_{0}, u_{0}\right), \quad t \geq t_{0} .
$$

The proof is complete.

Remark 3.3 Under the assumptions of Theorem 3.3, $g(t, u)$ satisfies $g(t, u)=L \cdot u$, where $L>0$. Then we obtain $D_{0}[\xi(t), \psi(t)] \leq D_{\sigma}\left[\xi_{0}, \psi_{0}\right] \exp (L t)$.

Theorem 3.4 Assume that $f \in C\left(\mathbb{R}^{+} \times C_{\sigma}, E^{d}\right), f$ maps bounded sets onto bounded sets and $f$ satisfies the following dissipative condition: there exist a continuous function $a: \mathbb{R}^{+} \rightarrow \mathbb{R}^{+}$ and $r \geq 0$ such that

$$
\left(x(t), f\left(t, x_{t}\right)\right)_{+} \leq a(t) D_{0}^{2}[x, \hat{0}]
$$

for every $t \geq 0$ and $x(t) \in E^{d}$ with $D_{0}[x, \hat{0}] \geq r$. Then the functional fuzzy differential equation (3.1) has a (ii)-solution on $\left[t_{0}, \infty\right]$.

Proof For $\left(t_{0}, \varphi_{0}\right) \in \mathbb{R}^{+} \times C_{\sigma}$ fixed, we consider the sets $I=\left[t_{0}, t_{0}+p\right)$ and $B_{\rho}=\left\{x \in C_{\sigma}\right.$ : $\left.D_{\sigma}[x, \hat{0}] \leq \rho\right\}$, where $p>0$ and $\rho>0$. Since $f$ maps bounded sets onto bounded sets, we deduce that there exists $M>0$ such that $D_{0}\left[f\left(t, x_{t}\right), \hat{0}\right] \leq M$ for every $(t, x) \in I \times B_{\rho}$. Therefore, there exists $\infty>\beta>t_{0}$ such that problem (3.1) has at least locally (ii)-solution $x(t)$ on some intervals $\left[t_{0}-\sigma, \beta\right]$. Let

$$
\mathcal{S}=\left\{x(t) \mid x(t) \text { is defined on } J_{x}=\left[t_{0}-\sigma, \eta_{x}\right) \text { and is the (ii)-solution to (3.1) }\right\} .
$$

Then $\mathcal{S} \neq \emptyset$. Next, we define a partial order $\lesssim$ on $\mathcal{S}$ as follows: $x \lesssim y$ if and only if $J_{x} \subseteq J_{y}$ and $x(t)=y(t)$ on $I_{x}$. Then the standard application of Zorn's lemma assures the existence of a maximal element $z$ in $(\mathcal{S}, \lesssim)$. The proof is complete if we show that $\eta_{z}=\infty$. Suppose that it is not true, so that $\eta_{z}<\infty$.

Step 1: We show that there exists $\gamma$ such that $D_{0}\left[z\left(t_{0}, \varphi_{0}\right)(t), \hat{0}\right] \leq \gamma$ for every $t \in J_{z}$. Define $\|z(t)\|=D_{0}\left[z\left(t_{0}, \varphi_{0}\right)(t), \hat{0}\right]$. Using Corollary 3.2 (see [10]), we have

$$
\frac{1}{2} \frac{d^{+}\|z(t)\|^{2}}{d t}=\left(z(t), D_{H}^{g} z(t)\right)_{+}, \quad t \in J_{z}
$$

that implies

$$
\frac{1}{2}\|z(t)\|^{2}=\frac{1}{2}\left\|z\left(t_{0}\right)\right\|^{2}+\int_{t_{0}}^{t}\left(z(s), D_{H}^{g} z(s)\right)_{+} d s, \quad t \in I_{z}
$$

and so

$$
\frac{1}{2}\|z(t)\|^{2}=\frac{1}{2}\left\|z\left(t_{0}\right)\right\|^{2}+\int_{t_{0}}^{t}\left(z(s), f\left(s, z_{s}\right)\right)_{+} d s, \quad t \in J_{z} .
$$


Now, we consider the sets $J_{1}=\left\{t \in J_{z}:\|z(t)\| \leq r\right\}$ and $J_{2}=J_{z} \bigvee_{1}$. Then we have that

$$
\left(z(t), f\left(t, z_{t}\right)\right)_{+} \leq a(t) D_{0}^{2}[z, \hat{0}]
$$

for every $t \in J_{2}$. On the other hand, since $f$ maps bounded sets onto bounded sets, there exists $M_{1}>0$ such that

$$
D_{0}\left[f\left(t, z_{t},\right), \hat{0}\right] \leq M_{1}, \quad \text { on } J_{z} \times B_{\rho},
$$

and so, by Theorem 3.2(i) (see [11]), it follows that

$$
\left(z(t), f\left(t, z_{t}\right)\right)_{+} \leq\|z(t)\| D_{0}\left[f\left(t, z_{t}\right), \hat{0}\right] \leq r M_{1}, \quad t \in J_{1} .
$$

From (3.16), (3.17) we obtain that

$$
(z(t), f(t, z(t)))_{+} \leq r M_{1}+a(t) D_{\sigma}^{2}[z, \hat{0}], \quad t \in J_{z}
$$

and so (3.15) becomes

$$
\begin{aligned}
\frac{1}{2}\|z(t)\|^{2} \leq & \left(\frac{1}{2}\left\|z\left(t_{0}\right)\right\|^{2}+r M_{1}\left(\eta_{z}-t_{0}\right)\right)+\int_{t_{0}}^{t} a(s) D_{\sigma}^{2}\left[z_{s}, \hat{0}\right] d s \\
\leq & \left(\frac{1}{2}\left\|z\left(t_{0}\right)\right\|^{2}+r M_{1}\left(\eta_{z}-t_{0}\right)\right) \\
& +\int_{t_{0}}^{t} a(s) \sup _{\theta \in[s-\sigma, s]} D_{0}^{2}[z(\theta), \hat{0}] d s, \quad t \in J_{z} .
\end{aligned}
$$

If we let $\xi(s)=\sup _{r \in[s-\sigma, s]} D_{0}^{2}[z(r), \hat{0}], s \in\left[t_{0}, t\right]$, then we have

$$
\frac{1}{2} \xi(t) \leq e(t)+\int_{t_{0}}^{t} a(s) \xi(s) d s
$$

By Gronwall's lemma, from inequality (3.18) we obtain that

$$
\|z(t)\|^{2} \leq \gamma^{2}=e(t)+\int_{t_{0}}^{t} e(s) a(s) \cdot \exp \left(\int_{s}^{t} a(r) d r\right) d s
$$

for every $t \in I_{z}$, where $e(t)=\left(\frac{1}{2}\left\|z\left(t_{0}\right)\right\|^{2}+r M_{1}\left(\beta_{z}-t_{0}\right)\right)$. Therefore, there exists $\gamma>0$ such that $D_{0}\left[z(t), \theta^{n}\right] \leq \gamma$ on $J_{z}$. We infer that $D_{0}\left[f\left(t, z_{t}\right), \hat{0}\right] \leq M_{z}$ for every $t \in J_{z}$.

Step 2: We show that $z(\cdot)$ is Lipschitzian on $I_{z}$. Indeed, for all $t_{1}, t_{2} \in I_{z}$ with $t_{1} \leq t_{2}$, we have

$$
\begin{aligned}
D_{0} & {\left[z\left(t_{1}, t_{0}, \varphi_{0}\right), z\left(t_{2}, t_{0}, \varphi_{0}\right)\right] } \\
& =D_{0}\left[\varphi(0) \ominus(-1) \int_{t_{0}}^{t_{1}} f\left(s, z_{s}\right) d s, \varphi(0) \ominus(-1) \int_{t_{0}}^{t_{2}} f\left(s, z_{s}\right) d s\right] \\
& \leq D_{0}\left[\int_{t_{1}}^{t_{2}} f\left(s, z_{s}\right) d s, \hat{0}\right] \leq \int_{t_{1}}^{t_{2}} D_{0}\left[f\left(s, z_{s}\right), \hat{0}\right] d s \leq M_{z}\left(t_{2}-t_{1}\right) .
\end{aligned}
$$


Therefore $z(\cdot)$ has a continuous extension $z^{*}(\cdot)$ on $\left[t_{0}, \eta_{z}\right]$. By continuity, we have

$$
z^{*}\left(\eta_{z}\right)=\varphi(0) \ominus(-1) \int_{t_{0}}^{\eta_{z}} f\left(s, z_{s}^{*}\right) d s
$$

This implies that $z^{*}(\cdot)$ is a solution of (3.1) on $\left[t_{0}-\sigma, \eta_{z}\right]$. Further, we consider FFDE with the new initial function at $t=\eta_{z}$

$$
D_{H}^{g} x(t)=f\left(t, x_{t}\right), \quad x\left(\eta_{z}\right)=\varphi\left(\eta_{z}-t_{0}\right)=\psi_{0} .
$$

Then the last FFDE has a solution $x^{*}(\cdot)$ on $\left[\eta_{z}-\sigma, \eta_{z}+q\right), q>0$. If we define

$$
z_{1}(t)= \begin{cases}z^{*}(t) & \text { for } t_{0}-\sigma \leq t \leq \eta_{z} \\ x^{*}(t) & \text { for } \eta_{z}-\sigma \leq t \leq \eta_{z}+q\end{cases}
$$

then it is clear that $z_{1}(t)$ is a solution of (3.1) on $\left[\eta_{z}-\sigma, \eta_{z}+q\right)$. This contradicts the maximality of $z(t)$ and hence $\eta_{z}=\infty$.

Theorem 3.5 Assume that the assumptions of Theorem 3.4 hold. In addition, we assume that $f \in C\left(\mathbb{R}^{+} \times C_{\sigma}, E^{d}\right)$ and $D_{0}[f(t, x), f(t, y)] \leq g\left(t, D_{\sigma}[x, y]\right)$ for $x, y \in C_{\sigma}, t \in \mathbb{R}^{+}$, where $g \in C\left(\mathbb{R}^{+} \times \mathbb{R}^{+}, \mathbb{R}^{+}\right), g(t, 0) \equiv 0$, and $u(t) \equiv 0$ is only a solution of

$$
\left\{\begin{array}{l}
\frac{d u}{d t}=g(t, u) \\
u\left(t_{0}\right)=0
\end{array}\right.
$$

for $t \geq t_{0}$. Then the functional fuzzy differential equation (3.1) has a unique (ii)-solution.

Proof It is easy to prove this theorem with the result of Theorem 3.3. Indeed, let $x(t)=$ $x\left(t_{0}, \varphi_{0}\right)(t)$ and $y(t)=y\left(t_{0}, \varphi_{0}\right)(t)$ be two solutions of (3.1) and (ii)-differentiable, we have: for $h>0$ small enough, there exist the Hukuhara differences $x(t-h) \ominus x(t), y(t-h) \ominus y(t)$. Now, for $t \in \mathbb{R}^{+}$, set $m(t)=D_{0}[x(t), y(t)]$ and noting that $m\left(t_{0}\right)=0$, we get

$$
D^{-} m(t)=\liminf _{h \rightarrow 0^{+}} \frac{1}{h}[m(t-h)-m(t)] \leq g\left(t, D_{\sigma}\left[x_{t}, x_{t}\right]\right),
$$

and so, by the comparison theorem for ordinary delay differential equations, we obtain

$$
D_{0}[x(t), y(t)] \leq r\left(t, t_{0}, 0\right), \quad t \geq t_{0}
$$

where $r\left(t, t_{0}, 0\right)$ is the solution of (3.19) on $\left[t_{0}, \infty\right)$. By assumption $r\left(t, t_{0}, 0\right)=0$, we obtain $x(t)=y(t)$ on $\left[t_{0}, \infty\right)$. The proof is complete.

\subsection{Illustrations}

Let us consider again the fuzzy functional differential equation with the initial value condition

$$
\begin{cases}D_{H}^{g} x(t)=f\left(t, x_{t}\right), & t \in J, \\ x(t)=\varphi\left(t-t_{0}\right), & t \in\left[-\sigma, t_{0}\right],\end{cases}
$$


where $f: I \times E^{1} \rightarrow E^{1}$. Let $[x(t)]^{\alpha}=[\underline{x}(t, \alpha), \bar{x}(t, \alpha)]$. By using Zadeh's extension principle, we obtain

$$
\left[f\left(t, x_{t}\right)\right]^{\alpha}=\left[\underline{f}_{-}\left(t, \alpha, \underline{x}_{t}(\alpha), \bar{x}_{t}(\alpha)\right), \bar{f}\left(t, \alpha, \underline{x}_{t}(\alpha), \bar{x}_{t}(\alpha)\right)\right]
$$

for $\alpha \in[0,1]$. By using Lemma 2.2, we have the following two cases.

If $x(t)$ is $\left[(\mathrm{i})\right.$-gH]-differentiable, then $\left[D_{H}^{g} x(t)\right]^{\alpha}=\left[\underline{x}^{\prime}(t, \alpha), \bar{x}^{\prime}(t, \alpha)\right]$ and $(3.20)$ is translated into the following functional differential system:

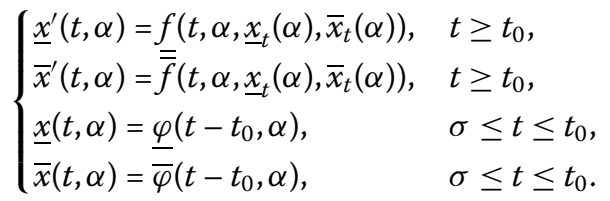

If $x(t)$ is [(ii)-gH]-differentiable, then $\left[D_{H}^{g} x(t)\right]^{\alpha}=\left[\bar{x}^{\prime}(t, \alpha), \underline{x}^{\prime}(t, \alpha)\right]$ and (3.20) is translated into the following functional differential system:

$$
\begin{cases}\bar{x}^{\prime}(t, \alpha)=f\left(t, \alpha, \underline{x}_{t}(\alpha), \bar{x}_{t}(\alpha)\right), & t \geq t_{0}, \\ \underline{x}^{\prime}(t, \alpha)=\overline{\bar{f}}\left(t, \alpha, \underline{x}_{t}(\alpha), \bar{x}_{t}(\alpha)\right), & t \geq t_{0}, \\ \underline{x}(t, \alpha)=\underline{\varphi}\left(t-t_{0}, \alpha\right), & \sigma \leq t \leq t_{0}, \\ \bar{x}(t, \alpha)=\bar{\varphi}\left(t-t_{0}, \alpha\right), & \sigma \leq t \leq t_{0} .\end{cases}
$$

Example 3.6 Let us consider the linear fuzzy functional differential equation under the generalized Hukuhara derivative

$$
\left\{\begin{array}{l}
D_{H}^{g} x(t)=-\lambda x\left(t-\frac{1}{2}\right), \\
x(t)=\varphi(t), \quad t \in\left[-\frac{1}{2}, 0\right]
\end{array}\right.
$$

where $[\varphi(t)]^{\alpha}=[\alpha-1,1-\alpha], \alpha \in[0,1], \lambda>0$. In this example we shall solve (3.23) on $[0,1]$.

Case 1: Considering [(i)-gH]-differentiability, we translate problem (3.23) into the following fuzzy delay system:

$$
\begin{cases}\underline{x}^{\prime}(t, \alpha)=-\lambda \bar{x}\left(t-\frac{1}{2}, \alpha\right), & t \geq 0, \\ \bar{x}^{\prime}(t, \alpha)=-\lambda \underline{x}\left(t-\frac{1}{2}, \alpha\right), & t \geq 0, \\ \underline{x}(t, \alpha)=\alpha-1, & -\frac{1}{2} \leq t \leq 0, \\ \bar{x}(t, \alpha)=1-\alpha, & -\frac{1}{2} \leq t \leq 0 .\end{cases}
$$

Solving fuzzy delay system (3.24) by using the method of steps, we obtain a unique (i)-solution to (3.23) defined on $[0,1]$ and it is of the form

$$
[x(t)]^{\alpha}=\left\{\begin{array}{cc}
{[(\alpha-1)(1+\lambda t),(1-\alpha)(1+\lambda t)]} & \text { for } t \in\left[0, \frac{1}{2}\right], \\
{\left[(\alpha-1)\left(\lambda t+\frac{\lambda^{2} t}{2}(t-1)+1+\frac{\lambda^{2}}{8}\right),\right.} & \\
\left.(1-\alpha)\left(\lambda t+\frac{\lambda^{2} t}{2}(t-1)+1+\frac{\lambda^{2}}{8}\right)\right] & \text { for } t \in\left[\frac{1}{2}, 1\right] .
\end{array}\right.
$$

The (i)-solution is illustrated in Figure 1. 
Figure 1 (i)-solution to (3.23) $(\lambda=0.5)$.

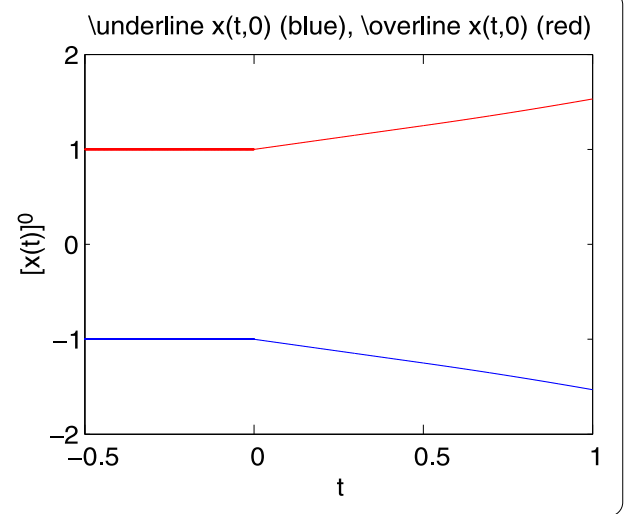

Figure 2 (ii)-solution to $(3.23)(\lambda=0.5)$.

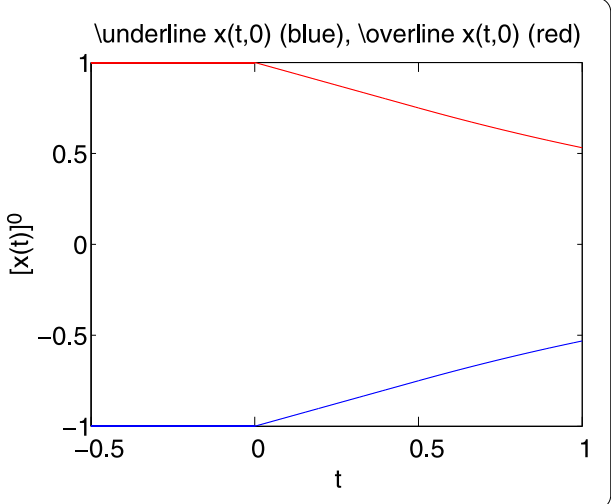

Case 2: Considering [(ii)-gH]-differentiability, we translate problem (3.23) into the following fuzzy delay system:

$$
\begin{cases}\bar{x}^{\prime}(t, \alpha)=-\lambda \bar{x}\left(t-\frac{1}{2}, \alpha\right), & t \geq 0 \\ \underline{x}^{\prime}(t, \alpha)=-\lambda \underline{x}\left(t-\frac{1}{2}, \alpha\right), & t \geq 0 \\ \underline{x}(t, \alpha)=\alpha-1, & -\frac{1}{2} \leq t \leq 0 \\ \bar{x}(t, \alpha)=1-\alpha, & -\frac{1}{2} \leq t \leq 0\end{cases}
$$

We obtain a unique (ii)-solution to (3.23) defined on $[0,1]$ and it is of the form

$$
[x(t)]^{\alpha}=\left\{\begin{array}{rr}
{[(\alpha-1)(1-\lambda t),(1-\alpha)(1-\lambda t)]} & \text { for } t \in\left[0, \frac{1}{2}\right], \\
{\left[(\alpha-1)\left(-\lambda t+\frac{\lambda^{2} t}{2}(t-1)+1+\frac{\lambda^{2}}{8}\right),\right.} & \\
\left.(1-\alpha)\left(-\lambda t+\frac{\lambda^{2} t}{2}(t-1)+1+\frac{\lambda^{2}}{8}\right)\right] & \text { for } t \in\left[\frac{1}{2}, 1\right] .
\end{array}\right.
$$

The (ii)-solution is illustrated in Figure 2.

\section{Sheaf fuzzy functional differential equations}

Let us consider again the sheaf initial value problem (SIVP) for a fuzzy functional differential equation (FDE) under the generalized Hukuhara derivative as follows:

$$
\begin{cases}D_{H}^{g} x(t)=f\left(t, x_{t}\right), & t \geq t_{0} \\ x(t)=\varphi\left(t-t_{0}\right)=\varphi_{0} \in H_{0}, & t_{0} \geq t \geq t_{0}-\sigma\end{cases}
$$

where $f \in C\left[I \times C_{\sigma}, E^{d}\right], H_{0} \subset C_{\sigma}$. 
Definition 4.1 The sheaf solution (or sheaf trajectory) of (4.1) under the generalized Hukuhara derivative gives at the time $t$ a set

$$
H_{t, x}=\left\{\begin{array}{l}
x(t)=\varphi\left(t-t_{0}\right) \in H_{0} \quad \text { for } t \in\left[t_{0}-\sigma, t_{0}\right], \\
x(t) \ominus_{g H} \varphi(0)=\int_{t_{0}}^{t} f(s, x(s)) d s,
\end{array}\right.
$$

where $x(t)$ is a solution of (4.1) for each $\varphi\left(t-t_{0}\right) \in H_{0} \subset C_{\sigma}$. We consider $H_{t, \widehat{x}}^{(i)}$ and $H_{t, \tilde{x}}^{(i i)}$ to be sheaf solutions of equation (4.1) in [(i)-gH]-differentiability type and [(ii)-gH]differentiability type, respectively, then by using Lemma 3.2, we have

$$
\begin{aligned}
H_{t, \widehat{x}}^{(i)} & = \begin{cases}x(t)=\varphi\left(t-t_{0}\right) \in H_{0} & \text { for } t \in\left[t_{0}-\sigma, t_{0}\right], \\
\widehat{x}(t)=\varphi(0)+\int_{t_{0}}^{t} f(s, \widehat{x}(s)) d s, & t \in I,\end{cases} \\
H_{t, \widehat{x}}^{(i i)} & = \begin{cases}x(t)=\varphi\left(t-t_{0}\right) \in H_{0} & \text { for } t \in\left[t_{0}-\sigma, t_{0}\right], \\
\widetilde{x}(t)=\varphi(0) \ominus(-1) \int_{t_{0}}^{t} f(s, \widetilde{x}(s)) d s, & t \in I .\end{cases}
\end{aligned}
$$

System (4.1) with its sheaf solutions is called a sheaf fuzzy problem. Suppose that $H_{t, x}, H_{t, y} \subset E^{d}$. Here are some useful notations:

(i) $d^{*}\left[H_{t, x}, H_{t, y}\right]=\sup _{x(t) \in H_{t, x}, y(t) \in H_{t, y}}\left\{D_{0}[x(t), y(t)]\right\}$;

(ii) $d^{*}\left[H_{t, x}, \hat{0}\right]=\left\|H_{t, x}\right\|_{*}=\sup _{x(t) \in H_{t, x}}\left\{D_{0}[x(t), \hat{0}]\right\}$;

(iii) $d_{\sigma}^{*}\left[H_{t, x}, H_{t, y}\right]=\sup _{t \in[-\sigma, 0]} d^{*}\left[H_{t, x}, H_{t, y}\right]$.

Definition 4.2 Let $H_{\cdot, x(\cdot)}: I \rightarrow \mathcal{H} \subset E^{d}$ be a fuzzy sheaf which is [(i)-gH]-differentiable (i.e., $x(t) \in H_{t, x}\left[(\mathrm{i})\right.$-gH]-differentiable for each $\left.\varphi\left(t-t_{0}\right) \in H_{0}\right)$. If $x$ and its derivative satisfy problem (4.1) for each $\varphi\left(t-t_{0}\right) \in H_{0}$, we say that $H_{t, x(t)}$ is a (i)-sheaf solution of problem (4.1).

Definition 4.3 Let $H_{, x(\cdot)}: I \rightarrow \mathcal{H} \subset E^{d}$ be a fuzzy sheaf such that [(i)-gH]-differentiable (i.e., $x(t) \in H_{t, x}\left[(\mathrm{i})\right.$-gH]-differentiable for each $\varphi\left(t-t_{0}\right) \in H_{0}$ ). If $x$ and its derivative satisfy problem (4.1) for each $\varphi\left(t-t_{0}\right) \in H_{0}$, we say that $H_{t, x(t)}$ is a (ii)-sheaf solution of problem (4.1).

Definition 4.4 A sheaf local solution $H_{, x(\cdot)}: I \rightarrow \mathcal{H} \subset E^{d}$ is unique if $d^{*}\left[H_{t, x}, H_{t, y}\right]=0$ for any $H_{, y(\cdot)}: I \rightarrow \mathcal{H} \subset E^{d}$, that is, a sheaf local solution to (4.1) on $I$.

In Section 3, under the generalized Lipschitz condition and dissipative condition, we proved the existence and uniqueness of the solution to both kinds of FFDE (3.1). In this section, we prove the existence and uniqueness of a sheaf solution to both kinds of sheaf fuzzy functional differential equation (SFFDE) (4.1) by using the results in Section 3.

Corollary 4.1 Let $\varphi\left(t-t_{0}\right) \in H_{0} \subset C_{\sigma}$ and suppose that $f:\left[t_{0}, t_{0}+p\right] \times S\left(x^{0}, \rho\right) \rightarrow E^{d}$ satisfies the condition: there exists a constant $L>0$ such that for every $\xi, \psi \in S\left(x^{0}, \rho\right)$ it holds

$$
D_{0}[f(t, \xi), f(t, \psi)] \leq L D_{\sigma}[\xi, \psi] .
$$

Moreover, there exists $M_{1}>0$ such that $D_{0}[f(t, \xi), \hat{0}] \leq M_{1}$. Then problem (4.1) has a unique sheaf solution for each case $([(i)$-gH]-differentiable or $[(i i)$-gH $]$-differentiable type $)$ on $\left[t_{0}, t_{0}+r\right]$, where $r=\min \left\{p, \rho / M_{1}, 1 / L, d\right\}$. 
Proof We prove the case of [(ii)-gH]-differentiability, the proof of the other case being similar. From the assumptions of this corollary and the result of Corollary 3.1, there exists at least locally (ii)-solution $\widetilde{x}(t)$ for (4.1) for each $\varphi\left(t-t_{0}\right) \in H_{0}$ on $J_{0}=\left[t_{0}, t_{0}+r\right]$, where $r=\min \left\{p, \rho / M_{1}, 1 / L, d\right\}$. Therefore $H_{t, \widetilde{x}}^{(i i)}$ is nonempty for every $\varphi\left(t-t_{0}\right) \in H_{0} \subset C_{\sigma}$. Now, we show that this (ii)-sheaf solution is unique. Indeed, let us assume that $\widetilde{x}(t), \widetilde{y}(t)$ are two solutions of (4.1). By definition of the solution we have $\widetilde{x}(t)=\widetilde{y}(t)$ if $t \in\left[t_{0}-\sigma, t_{0}\right]$. Note that for $t \in I$,

$$
d^{*}\left[H_{t, \widetilde{x}}^{(i i)}, H_{t, \widetilde{y}}^{(i i)}\right] \leq \int_{t_{0}}^{t} L d_{\sigma}^{*}\left[H_{s, \widetilde{x}_{s}}^{(i i)}, H_{s, \widetilde{y}_{s}}^{(i i)}\right] d s \leq L \int_{t_{0}}^{t} \sup _{\theta \in[s-\sigma, s]} d^{*}\left[H_{s, \widetilde{x}(\theta)}^{(i i)}, H_{s, \tilde{y}(\theta)}^{(i i)}\right] d s
$$

If we let $a(s)=\sup _{\tau \in[s-\sigma, s]} d^{*}\left[H_{s, \widetilde{x}(\tau)}^{(i i)}, H_{s, \tilde{y}(\tau)}^{(i i)}\right], s \in\left[t_{0}, t\right] \subset\left[t_{0}, t_{0}+r\right]$ and apply the Gronwall inequality, we obtain a more traditional estimation of the distance between two (ii)-sheaf solutions $H_{t, x}^{(i i)}$ and $H_{t, y}^{(i i)}$. Namely,

$$
d^{*}\left[H_{t, \widetilde{x}}^{(i i)}, H_{t, \widetilde{y}}^{(i i)}\right] \leq 0
$$

Therefore $d^{*}\left[H_{t, \widetilde{x}}^{(i i)}, H_{t, \tilde{y}}^{(i i)}\right]=0$. This proof is complete.

Example 4.1 Let us consider the linear sheaf fuzzy functional differential equation under two kinds of the Hukuhara derivative

$$
\left\{\begin{array}{l}
D_{H}^{g} x(t)=\lambda x(t-d) \\
x(t)=\varphi(t), \quad t \in[-d, 0]
\end{array}\right.
$$

where $\varphi(t) \in H_{0}=\left\{\varphi^{j}(t)=(j-t, j+1-t, j+2-t)\right\}_{j=0}^{\mathbb{N}} \subset C_{\sigma}, t \in[-d, 0], d>0, \lambda>0$. In this example, we shall solve $(4.4)$ on $[0, d]$.

Case 1: Considering [(i)-gH]-differentiability, we translate problem (4.4) into the following fuzzy delay system:

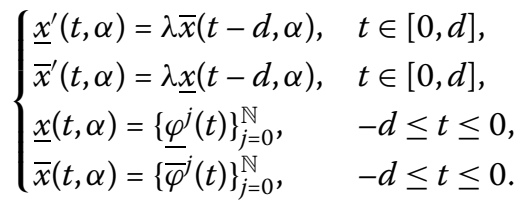

Solving fuzzy delay system (4.5) by using the method of steps, we obtain a unique (i)-sheaf solution to (4.4) defined on $[0, d]$ and it is of the form

$$
\begin{aligned}
H_{t, x}^{(i)}= & \left\{x^{j}(t)=\left(\lambda t\left[i-\frac{t}{2}+d\right]+i, \lambda t\left[i-\frac{t}{2}+d+1\right]+i+1,\right.\right. \\
& \left.\left.\lambda t\left[i+2-\frac{t}{2}+d\right]+i+2\right)\right\}_{j=0}^{\mathbb{N}} .
\end{aligned}
$$

The (i)-sheaf solution is illustrated in Figure 3. 


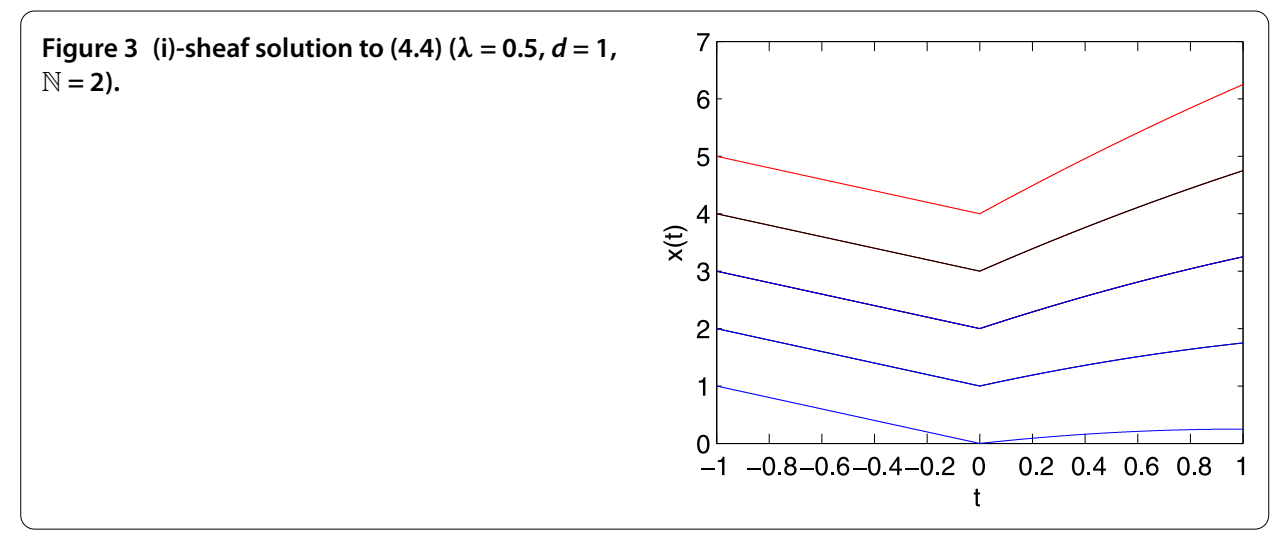

Figure 4 (ii)-sheaf solution to (4.4) $(\lambda=0.5, d=1$, $\mathbb{N}=2$ ).

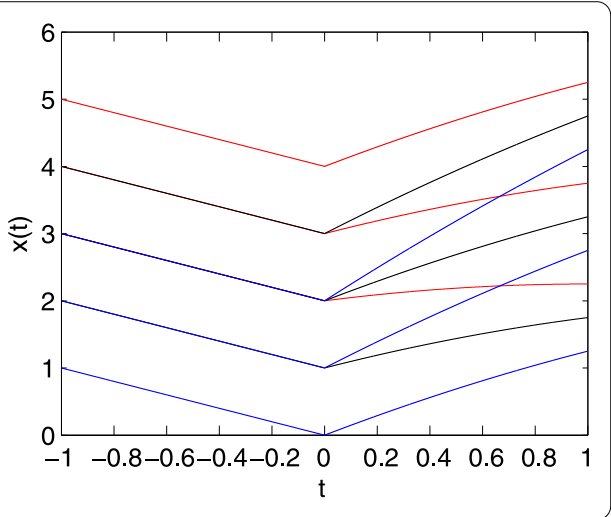

Case 2: Considering [(ii)-gH]-differentiability, we translate problem (3.23) into the following fuzzy delay system:

$$
\begin{cases}\underline{x}^{\prime}(t, \alpha)=\lambda \underline{x}(t-d, \alpha), & t \in[0, d], \\ \bar{x}^{\prime}(t, \alpha)=\lambda \bar{x}(t-d, \alpha), & t \in[0, d], \\ \underline{x}(t, \alpha)=\left\{\underline{\varphi}^{j}(t)\right\}_{j=0}^{\mathbb{N}}, & -d \leq t \leq 0, \\ \bar{x}(t, \alpha)=\left\{\bar{\varphi}^{j}(t)\right\}_{j=0}^{\mathbb{N}}, & -d \leq t \leq 0 .\end{cases}
$$

We obtain a unique (ii)-sheaf solution to (4.4) defined on $[0, d]$ and it is of the form

$$
\begin{aligned}
H_{t, x}^{(i i)}= & \left\{x^{j}(t)=\left(\lambda t\left[i+2-\frac{t}{2}+d\right]+i, \lambda t\left[i-\frac{t}{2}+d+1\right]+i+1,\right.\right. \\
& \left.\left.\lambda t\left[i-\frac{t}{2}+d\right]+i+2\right)\right\}_{j=0}^{\mathbb{N}} .
\end{aligned}
$$

The (ii)-sheaf solution is illustrated in Figure 4.

Suppose $x, y \in E^{1}$. We say that $x \leq y$ if and only if $\underline{x}(t, \alpha) \leq y(t, \alpha)$, and $\bar{x}(t, \alpha) \leq \bar{y}(t, \alpha)$, $\forall \alpha \in[0,1]$. We can also define the fuzzy interval $[x, y]=\left\{z \in E^{1}: x \leq z \leq y\right\}$. Letting $x, y \in$ $C\left([a, b], E^{1}\right)$ be two functions, we say that $x \leq y$ if $x(t) \leq y(t)$ for $t \in[a, b]$. Moreover, we define $[x(t), y(t)]=\left\{z \in C\left([a, b], E^{1}\right): x(t) \leq z(t) \leq y(t)\right\}$. 


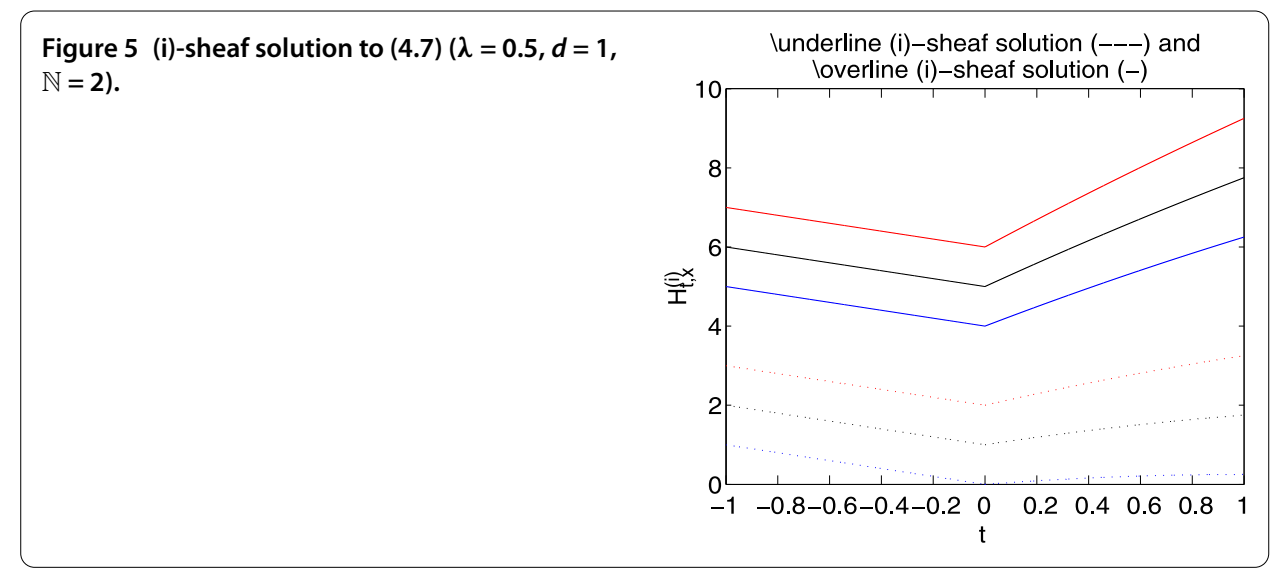

Figure 6 (ii)-sheaf solution to (4.7) $(\lambda=0.5, d=1$, $\mathbb{N}=2$ ).

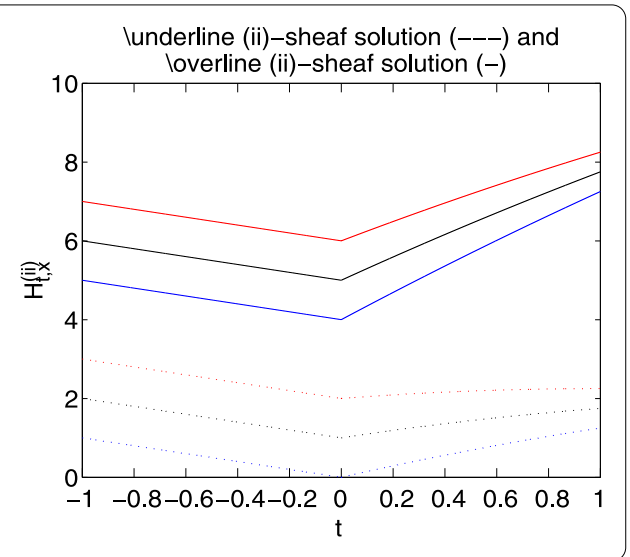

Example 4.2 Let us consider the linear sheaf fuzzy functional differential equation under two kinds of the Hukuhara derivative

$$
\left\{\begin{array}{l}
D_{H}^{g} x(t)=\lambda x(t-d) \\
x(t)=\varphi(t), \quad t \in[-d, 0]
\end{array}\right.
$$

where $\varphi(t) \in H_{0}=\left[\varphi^{1}(t), \varphi^{2}(t)\right] \subset C_{\sigma}, \varphi^{1}(t)=(-t, 1-t, 2-t), \varphi^{2}(t)=(4-t, 5-t, 6-t)$, $t \in[-d, 0], d>0, \lambda>0$. In this example, we shall solve (4.4) on [0,d].

One can obtain the (i)-sheaf solution and (i)-sheaf solution by using the methods as in the above examples.

The (i)-sheaf solution to (4.7) is defined on $[0, d]$ and it is of the form

$$
H_{t, x}^{(i)}=\left[x^{1}(t), x^{2}(t)\right]=\left[\begin{array}{c}
\left(\lambda t\left[-\frac{t}{2}+d\right], \lambda t\left[-\frac{t}{2}+d+1\right]+1, \lambda t\left[2-\frac{t}{2}+d\right]+2\right), \\
\left(\lambda t\left[4-\frac{t}{2}+d\right]+4, \lambda t\left[5-\frac{t}{2}+d\right]+5, \lambda t\left[6-\frac{t}{2}+d\right]+6\right)
\end{array}\right] .
$$

The (ii)-sheaf solution to (4.7) is defined on $[0, d]$ and it is of the form

$$
H_{t, x}^{(i i)}=\left[x^{1}(t), x^{2}(t)\right]=\left[\begin{array}{c}
\left(\lambda t\left[2-\frac{t}{2}+d\right], \lambda t\left[-\frac{t}{2}+d+1\right]+1, \lambda t\left[-\frac{t}{2}+d\right]+2\right) \\
\left(\lambda t\left[6-\frac{t}{2}+d\right]+4, \lambda t\left[5-\frac{t}{2}+d\right]+5, \lambda t\left[4-\frac{t}{2}+d\right]+6\right)
\end{array}\right] .
$$

In Figures 5 and 6, (i)-sheaf solution and (ii)-sheaf solution curves of (4.7) are given. 


\section{Competing interests}

The authors declare that they have no competing interests.

\section{Authors' contributions}

All authors contributed equally to the writing of this paper. All authors read and approved the final version of the manuscript.

\section{Author details}

'Faculty of Mathematics and Statistics, Ton Duc Thang University, No. 19 Nguyen Huu Tho Street, Tan Phong Ward, District 7, Ho Chi Minh City, Vietnam. ²Division of Computational Mathematics and Engineering, Institute for Computational Science, Ton Duc Thang University, Ho Chi Minh, Vietnam. ${ }^{3}$ Faculty of Mathematics and Computer Science, University of Science, VNU Ho Chi Minh City, Vietnam.

\section{Acknowledgements}

The authors would like to express their gratitude to the anonymous referees for their helpful comments and suggestions, which have greatly improved the paper. This research is funded by the Foundation for Science and Technology Development of Ton Duc Thang University (FOSTECT), website: http://fostect.tdt.edu.vn.

\section{Received: 26 August 2013 Accepted: 20 March 2014 Published: 27 May 2014}

\section{References}

1. Barros, LC, Bassanezi, RC, Tonelli, PA: Fuzzy modeling in population dynamics. Ecol. Model. 128, 27-33 (2000)

2. Buckley, JJ, Feuring, T: Fuzzy differential equations. Fuzzy Sets Syst. 110, $43-54$ (2000)

3. Mizukoshi, MT, Barros, LC, Chalco-Cano, Y, Roman-Flores, H, Bassanezi, RC: Fuzzy differential equations and the extension principle. Inf. Sci. 177, 3627-3635 (2007)

4. Prakash, P, Nieto, JJ, Kim, J-H, Rodríguez-López, R: Existence of solutions of fuzzy neutral differential equations in Banach spaces. Dyn. Syst. Appl. 14, 407-417 (2005)

5. Song, S, Wu, C: Existence and uniqueness of solutions to Cauchy problem of fuzzy differential equations. Fuzzy Sets Syst. 110, 55-67 (2000)

6. Wu, C, Song, S: Existence theorem to the Cauchy problem of fuzzy differential equations under compactness-type conditions. Inf. Sci. 108, 123-134 (1998)

7. Bede, B, Gal, SG: Generalizations of the differentiability of fuzzy-number-valued functions with applications to fuzzy differential equations. Fuzzy Sets Syst. 151, 581-599 (2005)

8. Chalco-Cano, Y, Román-Flores, H: On new solutions of fuzzy differential equations. Chaos Solitons Fractals 38 112-119(2008)

9. Nieto, JJ, Khastan, A, Ivaz, K: Numerical solution of fuzzy differential equations under generalized differentiability. Nonlinear Anal. Hybrid Syst. 3, 700-707 (2009)

10. Lupulescu, V: On a class of fuzzy functional differential equations. Fuzzy Sets Syst. 160(11), 1547-1562 (2009)

11. Lupulescu, V: Initial value problem for fuzzy differential equations under dissipative conditions. Inf. Sci. 178(23), 4523-4533 (2008)

12. Malinowski, MT: Interval differential equations with a second type Hukuhara derivative. Appl. Math. Lett. 24(12), 2118-2123 (2011)

13. Malinowski, MT: Random fuzzy differential equations under generalized Lipschitz condition. Nonlinear Anal., Real World Appl. 13(2), 860-881 (2012)

14. Malinowski, MT: Existence theorems for solutions to random fuzzy differential equations. Nonlinear Anal., Theory Methods Appl. 73(6), 1515-1532 (2010)

15. Malinowski, MT: On random fuzzy differential equations. Fuzzy Sets Syst. 160(21), 3152-3165 (2009)

16. Agarwal, RP, Lakshmikantham, V, Nieto, JJ: On the concept of solution for fractional differential equations with uncertainty. Nonlinear Anal. 72, 2859-2862 (2010)

17. Agarwal, RP, O'Regan, D, Lakshmikantham, V: Viability theory and fuzzy differential equations. Fuzzy Sets Syst. 151, 536-580 (2005)

18. Allahviranloo, T, Abbasbandy, S, Sedaghatfar, O, Darabi, P: A new method for solving fuzzy integro-differential equation under generalized differentiability. Neural Comput. Appl. 21, 191-196 (2012)

19. Allahviranloo, T, Salahshour, S, Abbasbandy, S: Solving fuzzy fractional differential equations by fuzzy Laplace transforms. Commun. Nonlinear Sci. Numer. Simul. 17(3), 1372-1381 (2012). doi:10.1016/j.cnsns.2011.07.005

20. Allahviranloo, T, Ghanbari, M, Haghi, E, Hosseinzadeh, A, Nouraei, R: A note on 'Fuzzy linear systems'. Fuzzy Sets Syst. $177,87-92(2011)$

21. Allahviranloo, T, Abbasbandy, S, Ahmady, N, Ahmady, E: Improved predictor corrector method for solving fuzzy initial value problems. Inf. Sci. 179, 945-955 (2009)

22. Allahviranloo, T, Kiani, NA, Motamedi, N: Solving fuzzy differential equations by differential transformation method. Inf. Sci. 179, 956-966 (2009)

23. Malinowski, MT: Second type Hukuhara differentiable solutions to the delay set-valued differential equations. Appl. Math. Comput. 218(18), 9427-9437 (2012)

24. Bede, B, Stefanini, L: Generalized differentiability of fuzzy-valued functions. Fuzzy Sets Syst. (2012). doi:10.1016/j.fss.2012.10.003

25. Stefanini, L, Bede, B: Generalized Hukuhara differentiability of interval-valued functions and interval differential equations. Nonlinear Anal. 71, 1311-1328 (2009)

26. Hoa, NV, Tri, PV, Dao, TT: Some global existence results and stability theorem for fuzzy functional differential equations. J. Intell. Fuzzy Syst. (accepted)

27. Lakshmikantham, V: Theory of fractional functional differential equations. Nonlinear Anal., Theory Methods Appl. 69, $3337-3343$ (2008) 
doi:10.1186/1687-1847-2014-156

Cite this article as: Tri et al.: Sheaf fuzzy problems for functional differential equations. Advances in Difference Equations 2014 2014:156

Submit your manuscript to a SpringerOpen ${ }^{\circ}$ journal and benefit from:

- Convenient online submission

- Rigorous peer review

- Immediate publication on acceptance

- Open access: articles freely available online

- High visibility within the field

- Retaining the copyright to your article

Submit your next manuscript at $\gg$ springeropen.com 